\title{
Bilayer graphene formed by passage of current through graphite: evidence for a three-dimensional structure
}

Article

Accepted Version

Harris, P. J. F., Slater, T. J. A., Haigh, S. J., Hage, F. S., Kepaptsoglou, D. M., Ramasse, Q. M. and Brydson, R. (2014) Bilayer graphene formed by passage of current through graphite: evidence for a three-dimensional structure. Nanotechnology, 25. 465601. ISSN 1361-6528 doi: https://doi.org/10.1088/0957-4484/25/46/465601 Available at https://centaur.reading.ac.uk/38041/

It is advisable to refer to the publisher's version if you intend to cite from the work. See Guidance on citing.

To link to this article DOI: http://dx.doi.org/10.1088/0957-4484/25/46/465601

Publisher: IOP

All outputs in CentAUR are protected by Intellectual Property Rights law, including copyright law. Copyright and IPR is retained by the creators or other copyright holders. Terms and conditions for use of this material are defined in the End User Agreement. 


\section{CentAUR}

Central Archive at the University of Reading

Reading's research outputs online 


\title{
Bilayer graphene formed by passage of current through graphite: evidence for a three-dimensional structure
}

\author{
Peter J.F. Harris ${ }^{1}$, Thomas J.A. Slater², Sarah J. Haigh², Fredrik S. Hage ${ }^{3}$, Despoina M. \\ Kepaptsoglou $^{3}$, Quentin M. Ramasse ${ }^{3}$ and Rik Brydson ${ }^{4}$ \\ ${ }^{1}$ Electron Microscopy Laboratory, Department of Chemistry, J.J. Thomson Building, University \\ of Reading, Whiteknights, Reading RG6 6AF, UK. \\ ${ }^{2}$ School of Materials, University of Manchester, Manchester M13 9PL, UK. \\ ${ }^{3}$ SuperSTEM Laboratory, SciTech Daresbury, Keckwick Lane, Daresbury, WA4 4AD, UK. \\ ${ }^{4}$ Institute for Materials Research, SPEME, University of Leeds, Leeds, LS2 9JT, U.K. \\ E-mail: p.j.f.harris@ reading.ac.uk
}

\begin{abstract}
The passage of an electric current through graphite or few-layer graphene can result in a striking structural transformation, but there is disagreement about the precise nature of this process.

Some workers have interpreted the phenomenon in terms of the sublimation and edge reconstruction of essentially flat graphitic structures. An alternative explanation is that the transformation actually involves a change from a flat to a three-dimensional structure. Here we describe detailed studies of carbon produced by the passage of a current through graphite which provide strong evidence that the transformed carbon is indeed three-dimensional. The evidence comes primarily from images obtained in the scanning transmission electron microscope using the technique of high-angle annular dark-field imaging, and from a detailed analysis of electron energy loss spectra. We discuss the possible mechanism of the transformation, and consider potential applications of "three-dimensional bilayer graphene".
\end{abstract}

KEYWORDS: Graphene, electron tomography, electron energy loss spectroscopy. 


\section{Introduction}

Over the past five years or so a number of groups have demonstrated that the passage of an electric current through graphite or few-layer graphene can produce a dramatic structural transformation [1-8]. This involves the formation of a carbon material with a highly irregular edge morphology, with many re-entrant structures and unusually-shaped features. Among the novel structures observed in the transformed carbon are nanotubes seamlessly joined to larger graphene regions. Some groups have suggested that the transformation occurs as a result of sublimation and edge reconstruction of flat graphene [1,2,6-8]. However, there are reasons to believe that the process actually involves a change from a flat to a three-dimensional structure [3-5]. In this paper, detailed studies of carbon nanostructures produced by the passage of a current through graphite are described. As well as conventional high resolution transmission electron microscopy (HRTEM), the techniques of high-angle annular dark-field (HAADF) and electron energy loss spectroscopy (EELS) in the scanning transmission electron microscope (STEM) are used to gain insights into the three-dimensional structure of the material. Tilting experiments, including full tomographic reconstructions, provide strong evidence for threedimensionality, and this is confirmed by detailed analyses of EELS results. We discuss possible mechanisms for the transformation of flat graphite into three-dimension structures, and suggest that the key to understanding this may lie in the edge terminations of graphene planes. We also consider possible applications for the new carbon, the most important of which may be in the area of supercapacitors.

\section{Methods}

In order to pass a current through small samples of graphite, we used a commercial arcevaporator, which is normally used for carbon-coating specimens for electron microscopy. In this 
unit the electrodes are graphite rods, one of which is thinned to a diameter of approximately 1.4 $\mathrm{mm}$ at the point of contact. Following evaporation, the thinned carbon rod was found to have slightly shortened, and a small deposit was formed in the area where the two rods made contact (see Supplementary Data for more details). This was collected and examined by TEM. Material collected from the fresh graphite rods was also imaged by TEM for comparison. Conventional TEM imaging was carried out using a JEOL 2010 microscope, with a point resolution of 0.19 $\mathrm{nm}$, operated at an accelerating voltage of $200 \mathrm{kV}$. Care was taken not to expose the carbon to an intense electron beam, to avoid irradiation damage.

We used the technique of HAADF-STEM imaging to establish the three-dimensionality of the bilayer graphene structures. Both tilt sequences and individual images have been analysed. Tilt series were first recorded using an aberration corrected FEI Titan G2 (S)TEM operating in HAADF-STEM mode at $80 \mathrm{kV}$ using a Fischione 2020 single tilt tomography holder. The tilt sequences were acquired using Xplore3D acquisition software at regular angular increments of $2^{\circ}$ in all cases. Reconstructions were performed using FEI's Inspect3D software with the SIRT algorithm using 20 iterations. Orthoslices and the isosurface visualisation were extracted using ImageJ.

Higher resolution HAADF-STEM images were recorded on an aberration corrected Nion UltraSTEM100 dedicated scanning transmission electron microscope operated at $60 \mathrm{kV}$ and with a source energy spread of $0.3 \mathrm{eV}[9,10]$. When using a convergence semi-angle of $30.6 \mathrm{mrad}$, this resulted in an electron probe size of $0.11 \mathrm{~nm}$ (full-width at half-maximum). Electron energy loss spectra were also collected. The EEL spectrometer collection semi-angle was 39.2 mrad. The relative $\pi^{*}$ peak intensity was extracted using a 3 Gaussian fitting procedure followed by normalisation to the integrated edge intensity over a $20 \mathrm{eV}$ window (from the edge onset), as 
described in reference 11 . This was done for every pixel in 2D spectral images. Relative $\pi^{*}$ intensity 'line scans' were then extracted from the spectral images as line profiles. All this was carried out using Gatan's Digital Micrograph (DM) software. The C-K edges were de-noised using principle component analysis implemented in the MSA plug-in for DM [12].

\section{Results}

A typical image of material from the fresh graphite rod is shown in Fig. 1(a). As expected, this consisted mainly of flat crystallites, ranging from a few $100 \mathrm{~nm}$ to about $5 \mu \mathrm{m}$ in size, containing up to 100 layers. The crystallites were often folded and buckled, and were covered with small amounts of finely-divided material. No nanotubes or other fullerene-related structures were seen in the fresh graphite. The carbon collected from the graphite rods following arcing contained some "normal" graphite, but this was accompanied by many regions which had a very different morphological appearance. A typical transformed area is shown in Fig. 1(b). As can be seen, the outline of the structure in this area of the material is more irregular than in the fresh graphite, with many curved and unusually-shaped features. The carbon is frequently found to be decorated with short nanotubes or nanoparticles, and in some cases, nanotube-like structures are seamlessly connected to the larger regions. In some areas, the transformed material had a much more regular appearance, as in Fig. 1(c). Here there is clearly some alignment between the edges of different regions of the carbon, and also with the nanotubes which are attached to the larger structures. High resolution images of the transformed carbon showed double edge contrast, as shown in Fig.

2. There are two possible interpretations of this contrast, firstly that the sample consists of flat, 4-layer graphene, and secondly that it is made up of hollow, three-dimensional structures with 
bilayer walls. We believe that the latter explanation is the correct one, even though energetics would seem to favour a flat structure.

Figure 3 shows a tilt sequence recorded using the FEI Titan microscope in HAADFSTEM mode. Recording these sequences proved to be challenging for a number of reasons. Even though a relatively low accelerating voltage was used, and the microscope's vacuum was extremely clean, some beam damage and contamination was evident after prolonged imaging. In addition, the highly irregular morphology of the samples caused problems, because the region of interest often became obscured by adjacent structures during tilting. Despite these difficulties, we were able to record a number of tilt sequences. Figure 3 shows a region tilted through an angle of $110^{\circ}$. The two structures labelled with arrows in the $-30^{\circ}$ image can be viewed throughout almost the whole sequence, and the ways in which their projected shapes change with tilt indicate that they are three-dimensional rather than flat. A video of this sequence can be seen in Supplementary Data. A further tilt sequence of a structure in the transformed carbon is shown in Fig. 4(a), with orthogonal slices through the reconstruction of the structure shown in Fig. 4(b). Figure $4(\mathrm{c})$ is a lower magnification image at $0^{\circ}$ tilt. The orthogonal slices in Fig. 4(b) show three dimensionality throughout. A video showing the isosurface visualisation of this structure is available in Supplementary Data. For comparison, a tilt series of a flat graphene structure, together with orthogonal slices through the reconstruction and a lower magnification image at $0^{\circ}$ tilt, is shown in Fig. 5. The reconstruction of this data was performed on the same angular range as in Fig. 4(a) $\left(-50^{\circ}\right.$ to $\left.+46^{\circ}\right)$, excluding images acquired out of this range. The orthogonal slice labelled YZ (Fig 5(b)), where $\mathrm{Z}$ is the direction down the optic axis, shows a clear distinction from that of the apparently three-dimensional structure (Fig 4(b)). 
We now discuss results from the Nion UltraSTEM100 instrument. We used this microscope to record a combination of HAADF-STEM images and EELS spectra. Figure 6(a) shows a HAADF-STEM image of a nanotube-like structure joined to a larger bilayer region. We determined the degree of curvature at the edge of the structure in Fig. 6(a) by estimating local changes in intensity of a specific feature of the carbon $\mathrm{K}$ ionization edge in the EELS spectrum, the so-called $\pi^{*}$ peak (a sharp and intense feature at an energy loss of $284 \mathrm{eV}$, corresponding to $\pi$ states delocalised across the graphene sheets). In the simple case of a planar anisotropic layered carbon material (e.g. graphite), the relative intensity of the $\pi^{*}$ peak compared to the overall integrated carbon K-edge intensity will increase as the spectral contribution of electrons that have experienced momentum transfer parallel to the crystallographic c axis increases (see e.g. Ref. 13). For the present bilayer material and experimental configuration (where, owing to the relatively large EELS collection angle, we predominantly record transitions to empty electron states oriented perpendicular to the electron beam direction) this translates into a significantly higher relative $\pi^{*}$ peak intensity when the electron probe is incident on an edge (where the graphitic planes are visibly aligned along the beam direction) than when the probe is located anywhere further into the structure (Fig. 6(b)). Away from the edge the EELS signal is dominated by electrons that have undergone momentum transfer parallel to the graphene layers and, in analogy to the case of graphite [13], the resulting spectrum is therefore referred to as 'inplane' in Fig. 6(b). A gradual increase in relative $\pi^{*}$ peak intensity would therefore indicate a gradual change in average orientation, which could in turn be interpreted in terms of structural curvature. Figure 6(c) shows a graph of the intensity of the $\pi^{*}$ peak (normalised to the integrated edge intensity over a $20 \mathrm{eV}$ window) as the probe is scanned over the region of the larger structure indicated in Fig. 6(a) and clearly shows this behaviour. The two graphene layers 
defining the edge of the structure are clearly resolved in the HAADF profile (Fig. 6(c)) and, comparing their position to the gradual change in relative $\pi^{*}$ peak intensity as the probe approaches the edge, this strongly suggests a gradual curvature, consistent with a threedimensional rather than a flat structure. In a flat structure with reconstructed or curled up edges one might expect a much more abrupt change in the relative $\pi^{*}$ peak intensity than that seen in Fig. 6(c).

We were also able to carry out HAADF and EELS analysis of regions throughout a tilt series, although the range of angles available was less than in the FEI Titan. Figure 7(a) shows a series of HAADF-STEM images of a distorted tube-like bilayer structure connected to a larger region tilted in 5 increments in a range of $\pm 17.2^{\circ}$ about the axis indicated on the image corresponding to a $0^{\circ}$ rotation. Throughout the entire range of tilts both carbon layers are resolved at the edge, whilst the general 'tube' shape and relative projected positions of attached distorted carbon change gradually. This clearly indicates a three-dimensional rather than a flat structure. Furthermore, Fig. 7(b) shows how the diameter of the 'tube' (as measured from the region indicated in (c) and (d)) decreases visibly when tilting from $-17.2^{\circ}$ (c) to $+17.2^{\circ}$ (d). This indicates an elliptical rather than purely circular 'tube' cross section. The $\mathrm{C}-\mathrm{K}$ edge was recorded from the extended region of the larger end of the 'tube' indicated for $0^{\circ}$ in Fig. 7(a). From the plot in Fig. 7(e), it is again quite clear that the relative $\pi^{*}$ peak intensity changes much more gradually than what might be expected in a flat material with abruptly curled up or reconstructed edges. Variation in the relative $\pi^{*}$ peak intensity at the extreme edge in Fig.7 (e) is mainly attributed to spectral noise increasing as the electron probe is 'falling off' the structure. Interestingly, there are barely any significant changes in the $\pi^{*}$ peak intensity within the central region of the measured area. This might be consistent with the elliptical cross section inferred 
from Fig. 7(b). A perfectly circular cross section would likely show a distinct effect on the $\pi^{*}$ peak intensity throughout the sample. Thus if taking the overall projected shape of the 'tube' into consideration, its structure might be described as a bilayer 'funnel' connected to a distorted bent 'tube', both with an elliptical cross section.

\section{Discussion}

Rather similar structures to those described in the present paper have been reported in studies by Jia and co-workers [1,8], by Huang et al. [2,6] and by Barreiro and colleagues [7], although in these experiments the transformations were observed using small graphene crystals inside a TEM. In each case, the authors of these papers concluded that the changes they observed involved sublimation and edge reconstruction of flat graphene rather than a transformation from two-dimensional to three-dimensional structures. The results presented in the present paper point to a different conclusion: that the structures formed are three-dimensional rather than flat, and that the transformation primarily involves a restructuring of the graphite rather than sublimation.

The mechanism of the restructuring is not entirely clear, but the key to understanding the process may lie in the edge structure of graphite. It is well established that graphite planes often have "closed" edges, so that the layers resemble folded sheets [14-17], as illustrated schematically in Fig. 8(a). The restructuring which occurs on passage of a current may simply involve an "opening" of the layers, as shown in Fig. 8(b). In an attempt to find evidence for this mechanism, we searched for fragments of graphite which displayed the early stages of the process, and which were oriented with the graphite sheets parallel to the beam. One of these regions is shown in Fig. 8(c). This clearly shows the opening of bilayer structures at the edges of 
the sheets. Such structures were not found in the fresh graphite. We do not rule out the possibility that sublimation/condensation may play some role in the structural transformation, but suggest that it is probably not the major factor. We note that recent in situ Joule heating experiments have shown that carbon sublimation can result in the formation of certain line defects in graphene [18].

It seems evident that the structures seen in the transformed carbon contain pentagonal and other non-six-membered rings, and that these are responsible for the observed faceting and reentrant features. One could speculate, therefore, that the opening process might be initiated at pentagonal rings which are believed to occur where zig-zag and armchair edges meet, as shown in Fig. 9 [19].

Another interesting question is why the transformed carbon structures are almost exclusively bilayer rather than monolayer. Most of the other studies of Joule heating of graphite have resulted in the formation of largely monolayer structures $[1,2,6]$. The reason for the differences between the present work and some of these other studies may lie in the nature of the starting materials. In our work, the starting material is a commercial synthetic graphite. In the work by Jia et al. the starting materials were graphitic nanoribbons produced by chemical vapor deposition [1], while Huang et al. used thinned HOPG (highly orientated pyrolytic graphite) $[2,6]$. It may be that in these forms of graphite the folding involves single layers rather than bilayers as in Fig. 8(a).

Clearly there is still much to learn about the transformation in graphite structure which can be induced by passage of an electric current. The results presented here suggest that the transformation involves an opening of graphite layers, which creates a 3-dimensional structure. However, it remains unclear why the passage of an electric current should have such an effect. 
More work on this phenomenon would be very useful. As well as helping to understand the processes described in the present paper, this might also lead to a better understanding of other processes such as the arc-synthesis of carbon nanotubes and the degradation behaviour of graphite electrodes.

Finally, it is interesting to discuss possible applications for the new carbon described here, which could be described as "three-dimensional bilayer graphene" (3DBG). One area for which the material appears particularly suitable is as an electrode material for supercapacitors. Compared with microporous carbon, which has traditionally been used in supercapacitors, 3DBG has relatively large pore sizes (typically of the order of a few 10s of nm), which would greatly facilitate the penetration of electrolyte ions into the pores. The structure of 3DBG also suggests it will have a much higher electrical conductivity than microporous carbon. Three-dimensional bilayer graphene also has advantages over three-dimensional graphene materials produced by assembling small fragments of graphene using aqueous methods (e.g. refs $20-22$ ). In these materials the 3D structure is reliant on weak forces such as van der Waals interactions, and they therefore lack mechanical strength. The graphene walls also have a tendency to "clump together", destroying the porosity. Three-dimensional bilayer graphene on the other hand is largely a continuous material in which the porosity is a result of pentagonal and other nonhexagonal carbon rings in the graphene structure, and would be expected to have far higher stability and superior mechanical properties.

As well as in supercapacitors, 3DBG may have applications in a range of other areas, including lithium ion batteries, hydrogen storage and catalysis.

\section{Acknowledgment}

SuperSTEM is the UK Engineering and Physical Sciences Research Council (EPSRC) National Facility for aberration-corrected STEM. P.J.F.H. acknowledges the EPSRC for time on the 
SuperSTEM facility and on the Manchester FEI Titan microscope. The FEI Titan G2 80-200 S/TEM is funded with support from HM Government (UK) and is associated with research capability of the Nuclear Advanced Manufacturing Research Centre. S.J.H. acknowledges funding support from the USA Defense Threat Reduction Agency (grant number HDTRA1-121-0013) and EPSRC (grant number EP/K016946/1). P.J.F.H. thanks Kazu Suenaga for discussions in the early stages of this work.

\section{References}

[1] Jia X T, Hofmann M, Meunier V, Sumpter, B G, Campos-Delgado, J, Romo-Herrera, J M, Son H, Hsieh Y P, Reina A, Kong J, Terrones M and Dresselhaus, M S 2009 Controlled formation of sharp zigzag and armchair edges in graphitic nanoribbons Science 323 1701-1705

[2] Huang J Y, Ding F, Yakobson B I, Lu P, Qi L and Li J 2009 In situ observation of graphene sublimation and multi-layer edge reconstructions Proceedings of the National Academy of Sciences of the United States of America 106 10103-10108

[3] Harris P J F 2009 Ultrathin graphitic structures and carbon nanotubes in a purified synthetic graphite Journal of Physics: Condensed Matter 21355009

[4] Harris P J F 2011 Structural transformation of graphite by arc-discharge Philosophical Magazine 91 2355-2363

[5] Harris P J F 2012 Hollow structures with bilayer graphene walls Carbon 50 3195-3199

[6] Qi L, Huang J Y, Feng J and Li J 2010 In situ observations of the nucleation and growth of atomically sharp graphene bilayer edges Carbon 48 2354-2360

[7] Barreiro A, Börrnert F, Rümmeli M H, Büchner B, Vandersypen L M K 2012 Graphene at high bias: Cracking, layer by layer sublimation, and fusing Nano Letters 12 1873-1878

[8] Cruz-Silva E, Jia X T, Terrones H, Sumpter B G, Terrones M, Dresselhaus M S and Meunier V 2013 Edge-edge interactions in stacked graphene nanoplatelets ACS NANO 7 2834-2841

[9] Krivanek O L, Dellby N, Murfitt M F, Chisholm M F, Pennycook T J, Suenaga K and Nicolosi V 2010 Gentle STEM: ADF imaging and EELS at low primary energies Ultramicroscopy $110935-945$

[10] Krivanek O L, Corbin G J, Dellby N, Elston B F, Keyse R J, Murfitt M F, Own C S, Szilagyi Z S and Woodruff J W 2008 An electron microscope for the aberration-corrected era Ultramicroscopy 108 179-195

[11] Zhang Z L, Brydson R, Aslam Z, Reddy S, Brown A, Westwood A and Rand B 2011 Investigating the structure of non-graphitising carbons using electron energy loss spectroscopy in the transmission electron microscope Carbon 49 5049-5063 
[12] Watanabe M, Kanno M, Ackland D W, Kiely C J and Williams D B Applications of electron energy-loss spectrometry and energy filtering in an aberration-corrected JEM2200FS STEM/TEM 2007 Microscopy and Microanalysis 13 1264-1265

[13] Batson P E Carbon 1s near-edge-absorption fine structure in graphite 1993 Physical Review B 48 2608-2610

[14] Buseck P R, Huang B J and Keller L P 1987 Electron-microscope investigation of the structures of annealed carbons Energy and Fuels 1 105-110

[15] Ugarte D 1992 Morphology and structure of graphitic soot particles generated in arcdischarge $\mathrm{C}_{60}$ production Chem. Phys. Lett. 198 596-602

[16] Murayama H and Maeda T 1990 A novel form of filamentous graphite Nature $345791-$ 793

[17] Gogotsi Y, Libera J A, Kalashnikov N and Yoshimura M 2000 Graphite polyhedral crystals Science $290317-320$

[18] Chen J H, Autès G, Alem N, Gargiulo F, Gautam A, Linck M, Kisielowski C, Yazyev O V, Louie S G and Zettl A 2014 Controlled growth of a line defect in graphene and implications for gate-tunable valley filtering Phys. Rev. B 89, 121407(R)

[19] Liu Z, Suenaga K, Harris P J F and Iijima S 2009 Open and closed edges of graphene layers Phys. Rev. Lett. 102015501

[20] Chen W F and Yan L F 2011 In situ self-assembly of mild chemical reduction graphene for three-dimensional architectures Nanoscale 3 3132-3137

[21] Ji C C, Xu M W, Bao S J, Cai C J, Lu Z J, Chai H, Yang F and Wei H 2013 Selfassembly of three-dimensional interconnected graphene-based aerogels and its application in supercapacitors Journal of Colloid and Interface Science 407 416-424

[22] Tan Z, Ohara S, Abe H and Naito M 2014 Synthesis and processing of graphene hydrogels for electronics applications RSC Advances 4 8874-8878

FIGURES

Figure 1. (a) Transmission electron micrograph of carbon from fresh graphite rod. (b)

Region with disordered structure following passage of current. (c) Region with more aligned structure following passage of current.

Figure 2. High resolution TEM image of transformed carbon showing double edge contrast. 
Figure 3. HAADF-STEM images of region of transformed carbon, recorded at tilt angles from $+60^{\circ}$ to $-50^{\circ}$. Tilt axis indicated in top left image. Arrows in bottom left image indicate two apparently three-dimensional structures.

Figure 4. HAADF-STEM images of apparent three-dimensional structure in transformed carbon. (a) Cropped HAADF images recorded at tilt angles from $-50^{\circ}$ to $+46^{\circ}$. (b) Orthogonal slices through a SIRT reconstruction. (c) Lower magnification HAADF image of area from which tilt sequence was acquired.

Figure 5. HAADF-STEM images of a flat edge region in transformed carbon. (a) Cropped HAADF images at tilt angles from $-50^{\circ}$ to $+66^{\circ}$. (b) Orthogonal slices through a SIRT reconstruction. (c) Lower magnification HAADF image of area from which tilt sequence was acquired.

Figure 6. (a) HAADF-STEM image of region in which nanotube-like structure is joined to a larger region, (b) 'In-plane' and edge C-K edges from opposite ends of the region indicated in (a) showing the distinct difference in relative $\pi^{*}$ peak intensity characteristic of anisotropic carbons. (c) Plot showing a gradual increase in the relative $\mathrm{C}-\mathrm{K}$ edge $\pi^{*}$ peak contribution with increasing proximity to the edge from the region of the larger structure indicated in (a). The solid line fit to the data points is provided as a guide to the eye.

Figure 7. (a) HAADF-STEM images of a distorted tube-like structure recorded at a range of tilt angles. The axis of rotation is indicated. (b) 'Tube' diameter at different angles measured along the direction indicated in (c) and (d). (e) Plot showing a gradual increase in the relative contribution of the $\pi^{*}$ peak as the incident electron beam 
approaches the edge of the structure in (a). The solid line is provided as a guide to the eye, and is calculated by a smoothing of the discrete $\pi^{*}$ peak intensity data points.

Figure 8. (a), (b) Schematic illustration showing suggested mechanism for transformation of folded graphene sheets into hollow structure, (c) HRTEM image of graphitic region edge-on, apparently showing early stages of transformation.

Figure 9. Drawings of folded monolayer graphene, showing region where zig-zag edge meets armchair edge. For simplicity, only the upper layer is shown in the planview drawing. Arrow indicates position of pentagonal ring. 

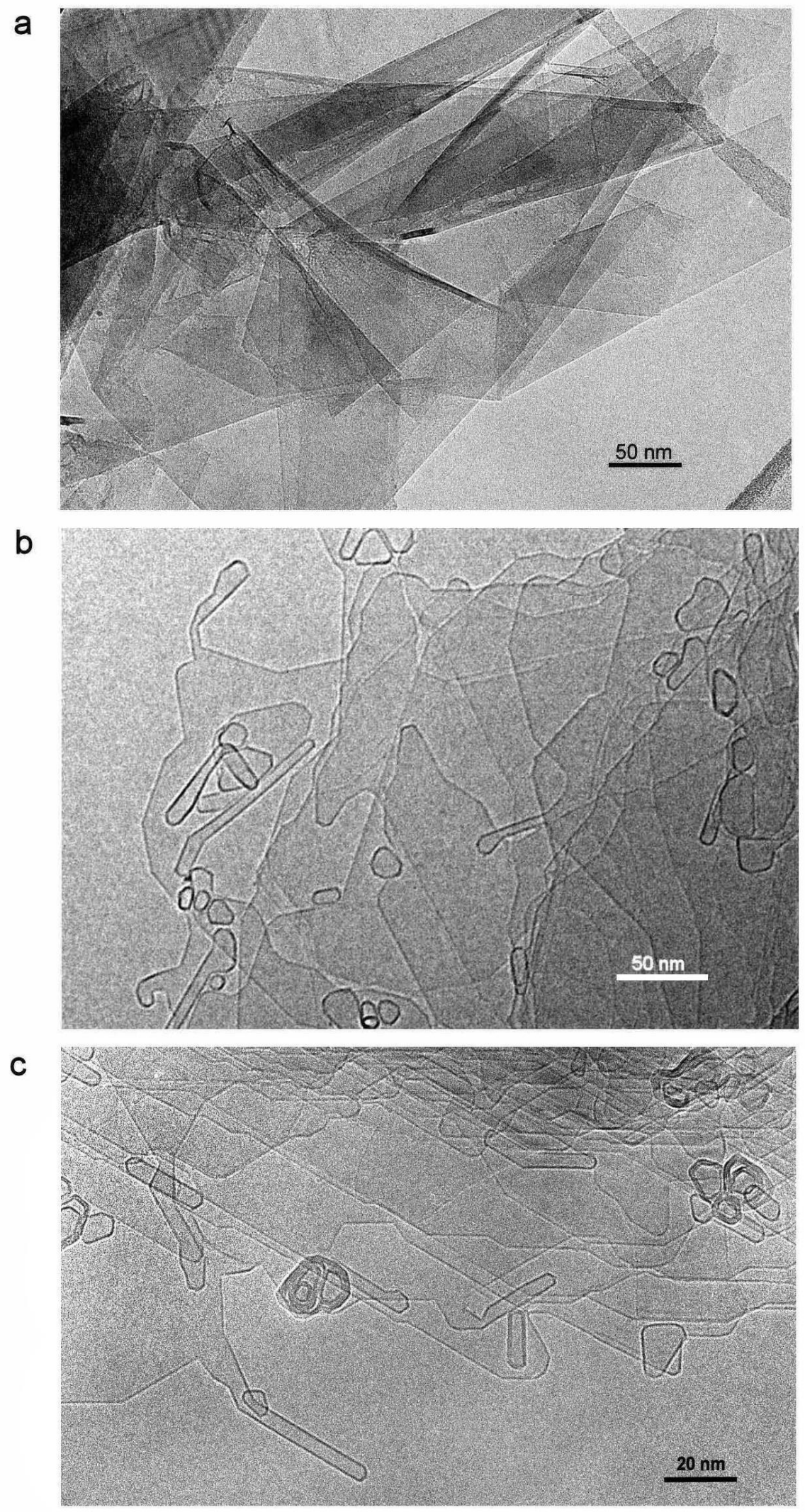

Figure 1 


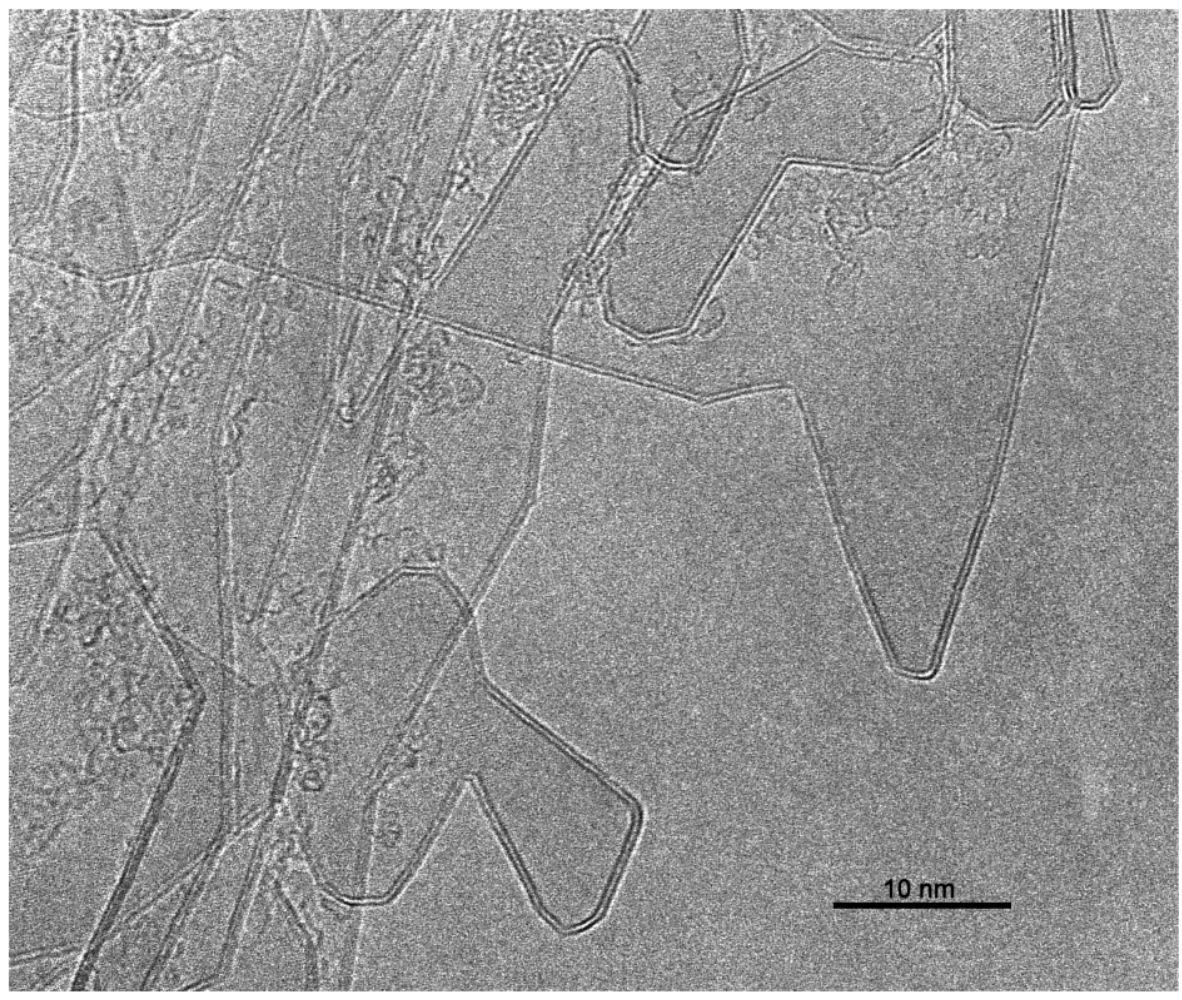

Figure 2 

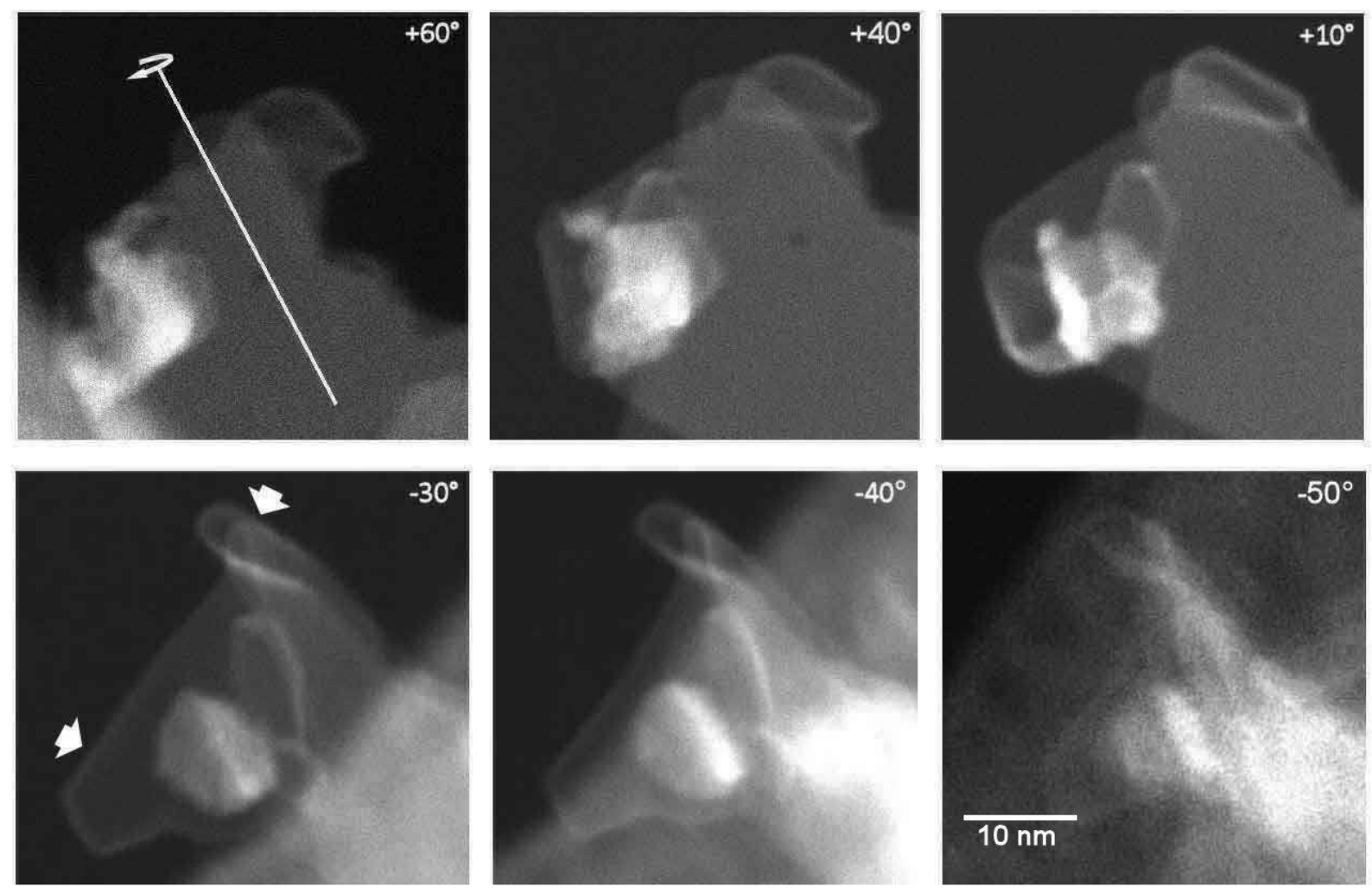

Figure 3
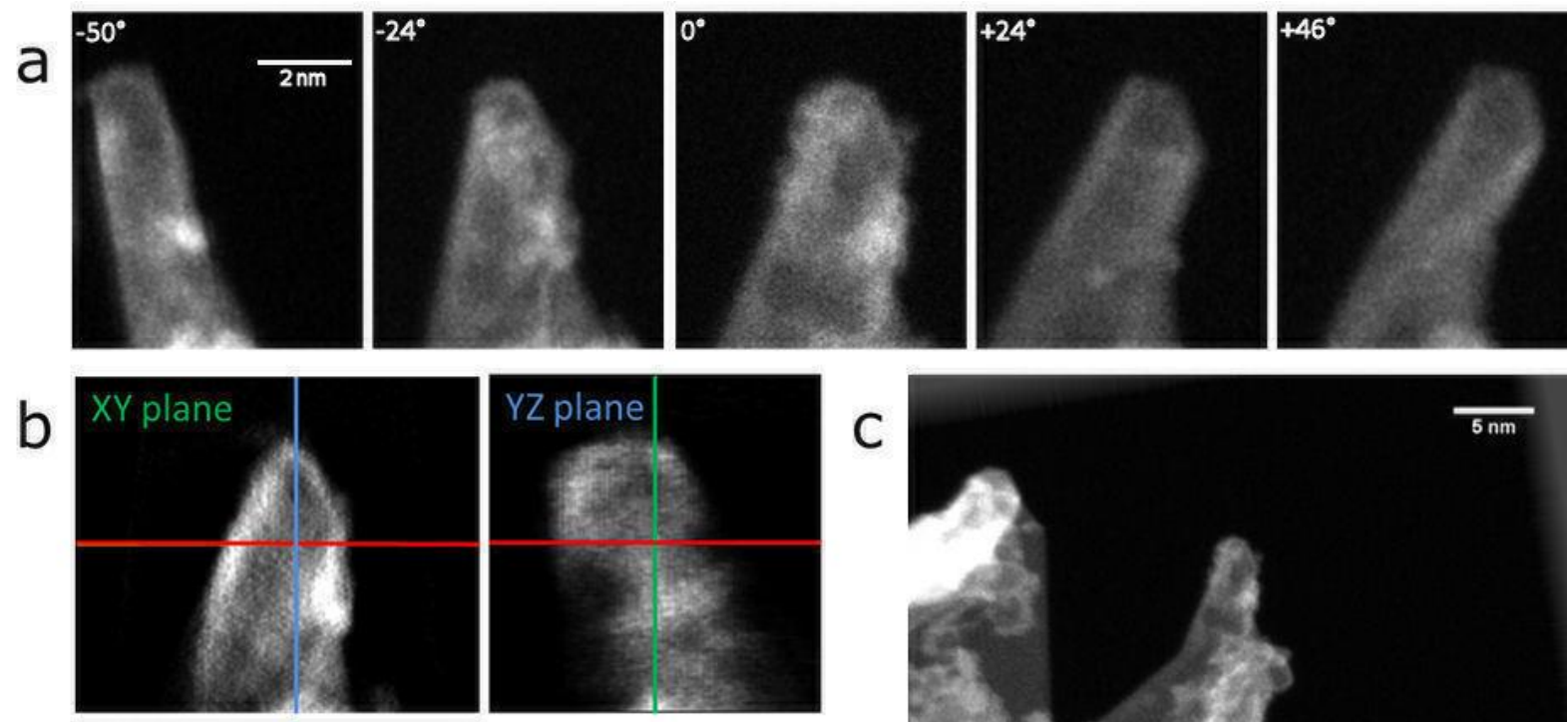

C
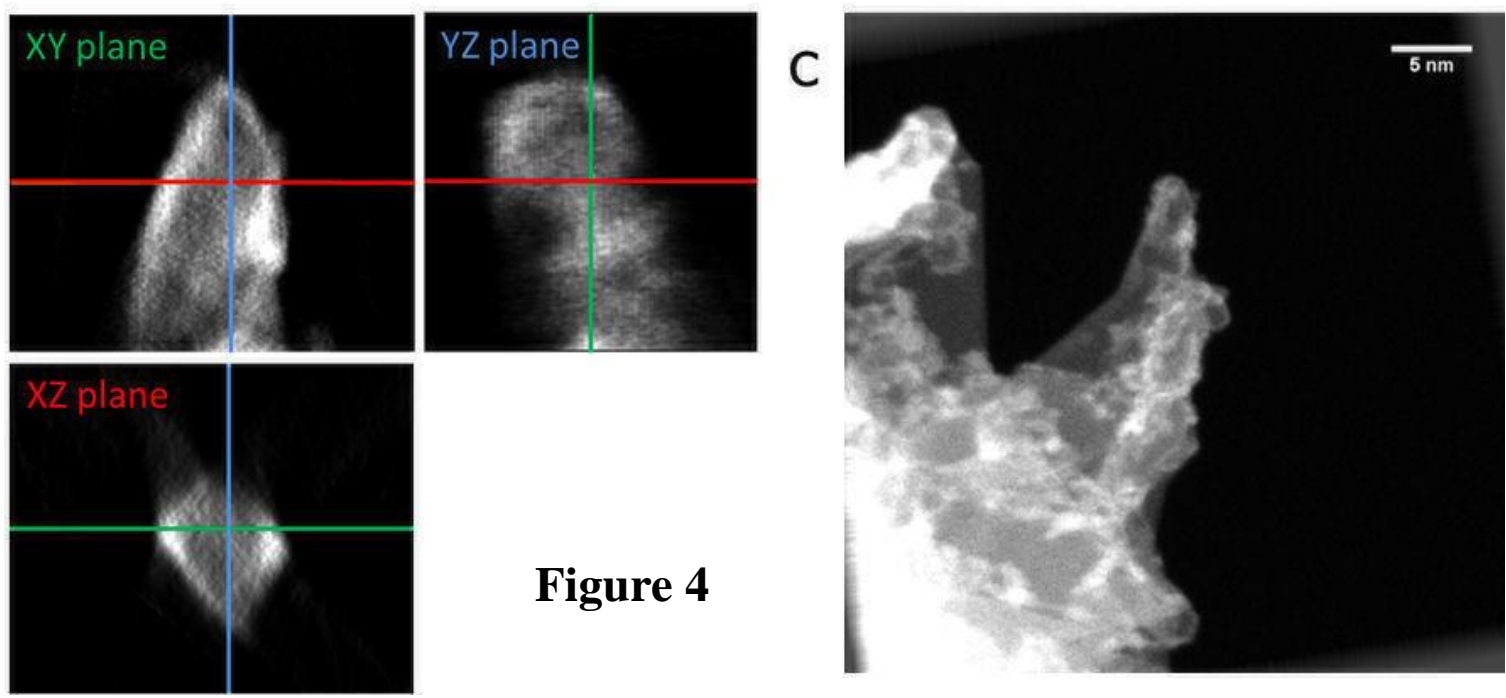

Figure 4 

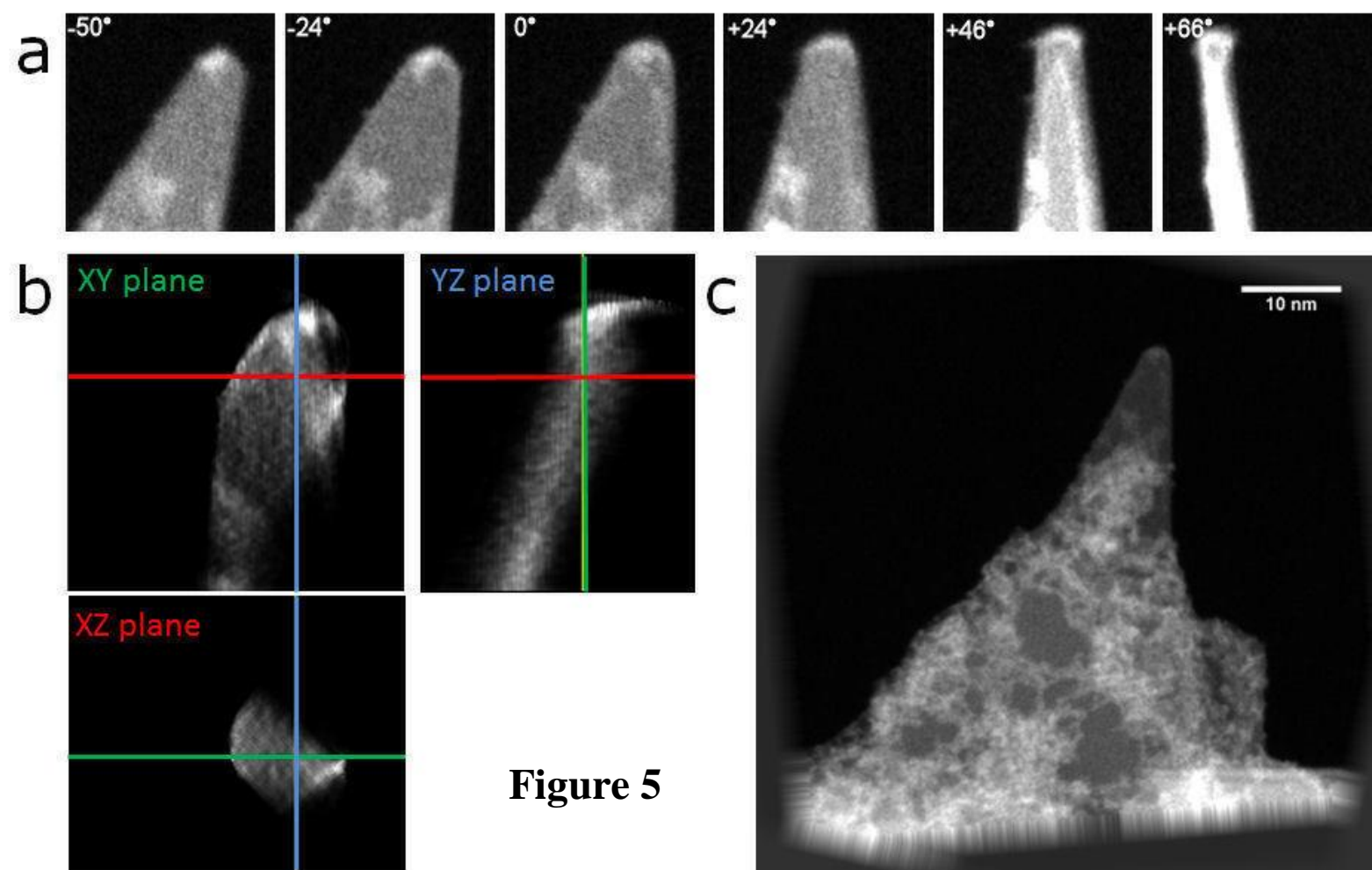

Figure 5

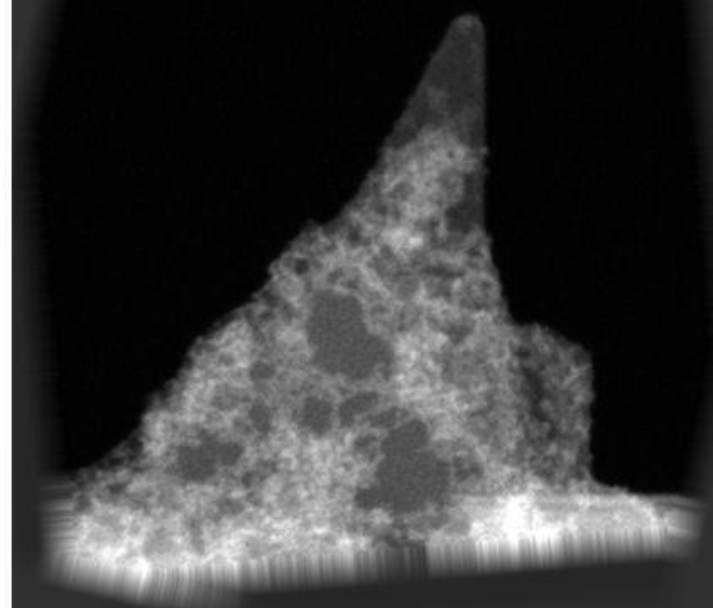



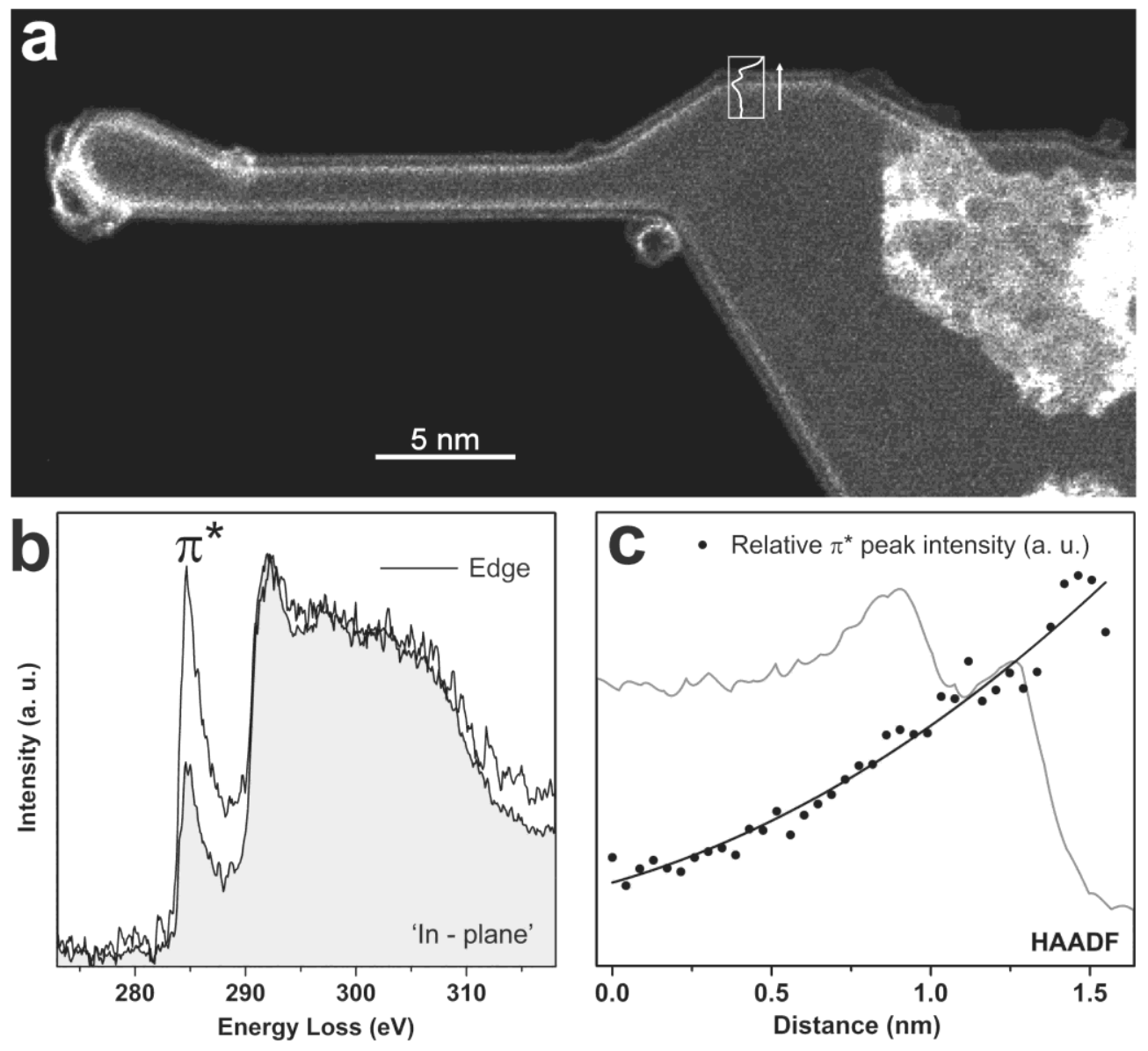

Figure 6 


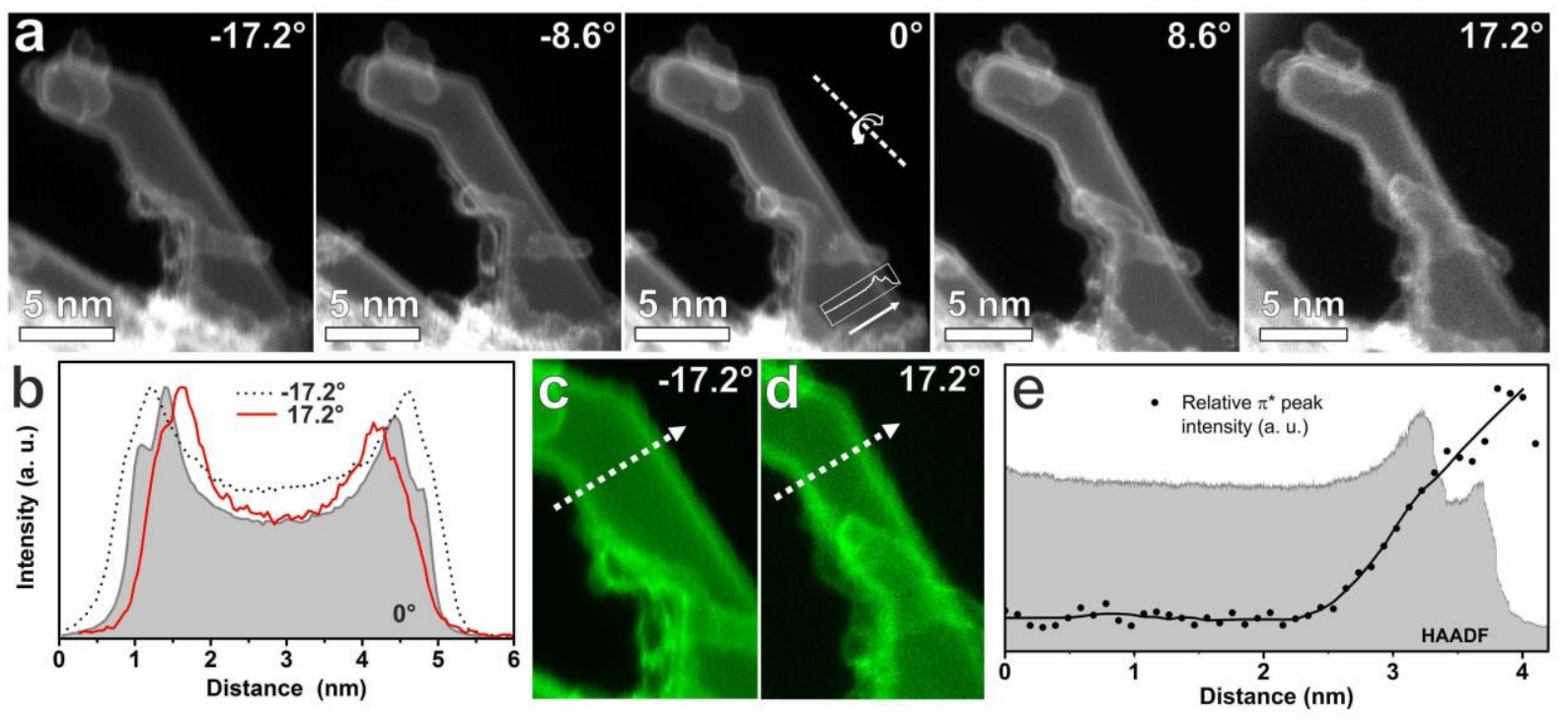

Figure 7 
a

b

$\longrightarrow \rightarrow$

c

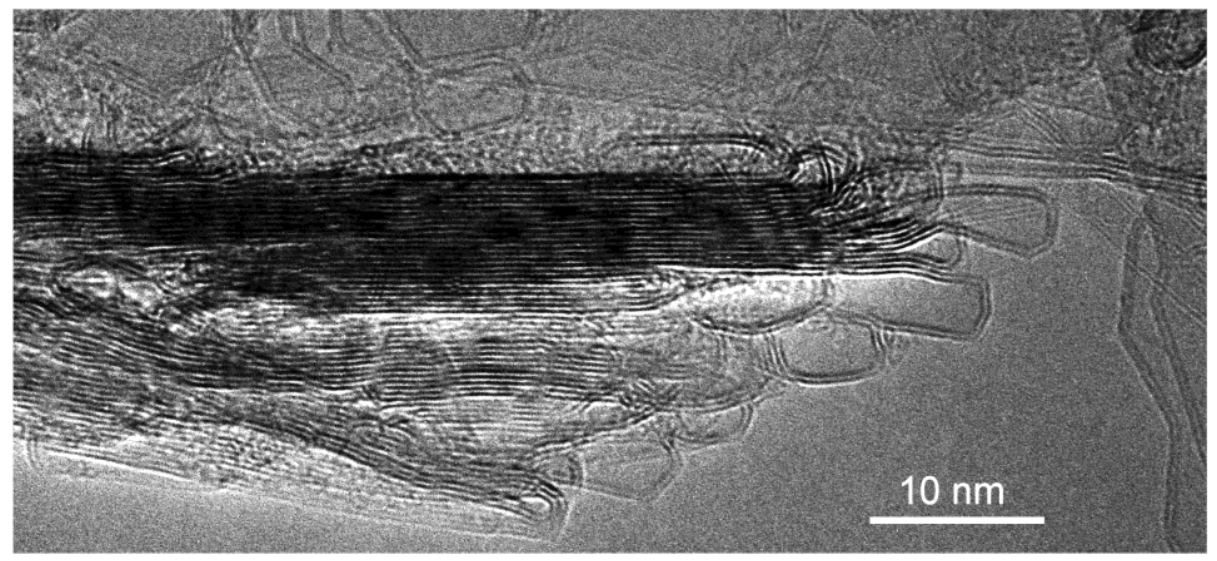

Figure 8
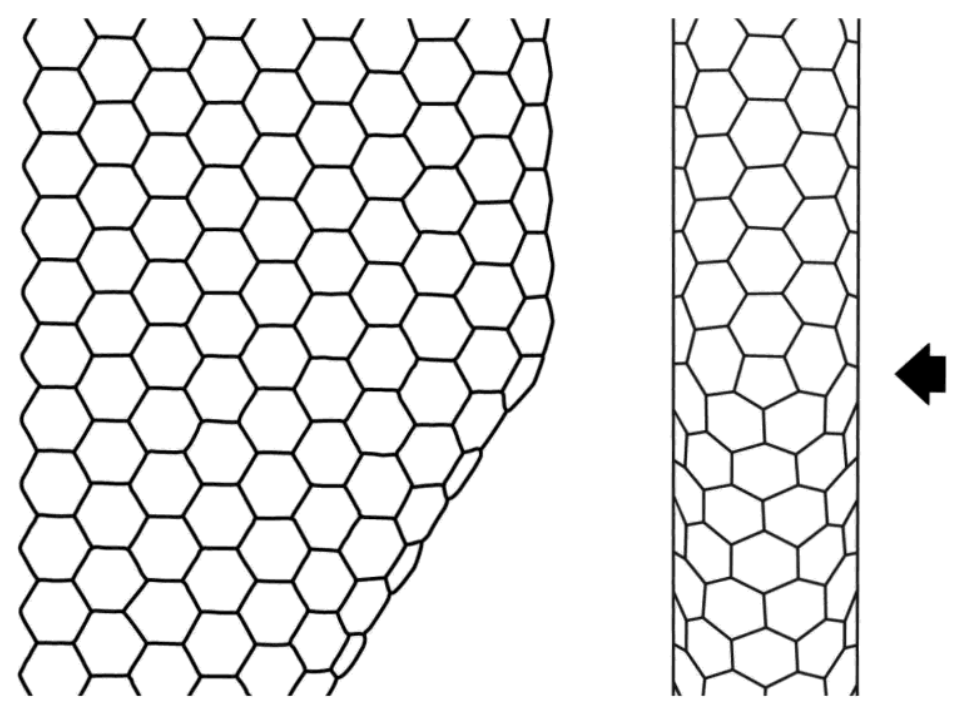

Figure 9 